\title{
Intraoperative awareness risk, anesthetic sensitivity, and anesthetic management for patients with natural red hair: a matched cohort study
}

\section{Le risque d'éveil peropératoire, la sensibilité aux anesthésiques et la gestion anesthésique chez les patients naturellement roux: une étude de cohorte appariée}

\author{
Stephen C. Gradwohl, MD • Amrita Aranake, MD • Arbi Ben Abdallah, PhD • \\ Paul McNair, BS · Nan Lin, PhD • Bradley A. Fritz, BS • Alex Villafranca, MS • \\ David Glick, MD • Eric Jacobsohn, MBChB · George A. Mashour, MD, PhD • \\ Michael S. Avidan, MBBCh
}

Received: 29 May 2014/ Accepted: 16 December 2014/Published online: 14 February 2015

(C) Canadian Anesthesiologists' Society 2015

\begin{abstract}
Purpose The red-hair phenotype, which is often produced by mutations in the melanocortin-1 receptor gene, has been associated with an increase in sedative, anesthetic, and analgesic requirements in both animal and human studies. Nevertheless, the clinical implications of
\end{abstract}

This article will be accompanied by an editorial. Please see Can J Anesth 2015; 62: this issue.

Author contributions Stephen Gradwohl, Amrita Aranake, Arbi Ben Abdallah, David Glick, George A. Mashour, and Michael S. Avidan designed the study. Alex Villafranca, David Glick, and Michael S. Avidan enrolled patients. Alex Villafranca and David Glick provided data. Amrita Aranake, Paul McNair, and Bradley A. Fritz entered data. Stephen Gradwohl, Amrita Aranake, Paul McNair, and Michael S. Avidan organized data. Stephen Gradwohl, Amrita Aranake, Arbi Ben Abdallah, and Michael S. Avidan conducted analyses. Stephen Gradwohl, Amrita Aranake, Arbi Ben Abdallah, Eric Jacobsohn, George A. Mashour, and Michael S. Avidan wrote the manuscript. Stephen Gradwohl, Amrita Aranake, Arbi Ben Abdallah, Paul McNair, Nan Lin, Bradley A. Fritz, Alex Villafranca, David Glick, Eric Jacobsohn, George A. Mashour, and Michael S. Avidan revised the manuscript. Stephen Gradwohl and Amrita Aranake contributed equally to this manuscript and are joint lead authors. Nan Lin conducted statistical analyses (especially the mixedeffects model). Bradley A. Fritz conducted statistical analyses (especially Kaplan-Meier analyses)

S. C. Gradwohl, MD - A. Aranake, MD

A. B. Abdallah, $\mathrm{PhD}$. P. McNair, BS

B. A. Fritz, BS · M. S. Avidan, MBBCh ( $\square)$.

Department of Anesthesiology, Washington University in Saint

Louis, School of Medicine, Campus Box 8054, 660 S. Euclid

Ave., Saint Louis, MO 63110, USA

e-mail: avidanm@anest.wustl.edu this phenomenon in red-haired patients undergoing surgery are currently unknown.

Methods In a secondary analysis of a prospective trial of intraoperative awareness, red-haired patients were identified and matched with five control patients, and the relative risk for intraoperative awareness was determined. Overall anesthetic management between groups was compared using Hotelling's $T^{2}$ statistic. Inhaled anesthetic requirements were compared between cohorts by evaluating the relationship between end-tidal anesthetic concentration and the bispectral index with a linear mixedeffects model. Time to recovery was compared using Kaplan-Meier analysis, and differences in postoperative pain and nausea/vomiting were evaluated with Chi square tests.

Results A cohort of 319 red-haired patients was matched with 1,595 control patients for a sample size of 1,914. There were no significant differences in the relative risk of intraoperative awareness (relative risk $=1.67 ; 95 \%$ confidence interval 0.34 to 8.22), anesthetic management, recovery times, or postoperative pain between red-haired patients and control patients. The relationship between pharmacokinetically stable volatile anesthetic concentrations and bispectral index values differed

N. Lin, PhD

Division of Biostatistics, Washington University in Saint Louis, School of Medicine, Saint Louis, MO, USA

A. Villafranca, MS · E. Jacobsohn, MBChB Department of Anesthesia and Perioperative Medicine, University of Manitoba, Winnipeg, MB, Canada 
significantly between red-haired patients and controls $(P<0.001)$, but without clinical implications.

Conclusion There were no demonstrable differences between red-haired patients and controls in response to anesthetic and analgesic agents or in recovery parameters. These findings suggest that perioperative anesthetic and analgesic management should not be altered based on selfreported red-hair phenotype.

\section{Résumé}

Objectif Le phénotype "roux» qui est souvent le résultat de mutations du gène codant pour le récepteur de la mélanocortine-1 a été associé à une augmentation des besoins en sédatifs, anesthésiques et analgésiques dans les études humaines et animales. Néanmoins, les implications cliniques de ce phénomène pour les patients roux subissant une chirurgie sont actuellement inconnues. Méthodes Des patients roux ont été identifiés dans une analyse secondaire d'une étude prospective d'éveil peropératoire et ils ont été appariés à cinq patients témoins; leur risque relatif d'éveil peropératoire a été déterminé. La gestion anesthésique globale a été comparée entre les groupes en utilisant la méthode statistique $T^{2}$ de Hotelling. Les besoins d'anesthésiques inhalés ont été comparés entre les cohortes par l'évaluation des relations entre les concentrations d'anesthésiques en fin de respiration et l'indice bispectral avec un modèle linéaire d'effets mélangés. Le délai de récupération a été comparé en utilisant la méthode d'analyse de Kaplan-Meier et les différences concernant la douleur postopératoire et les nausées/vomissements ont été évaluées par des tests du Chi carré.

Résultats Une cohorte de 319 patients roux a été appariée à 1595 patients témoins dans un échantillon total de 1914 patients. Il n'y a pas eu de différences statistiquement significatives sur le risque relatif d'éveil peropératoire (risque relatif: 1,67; intervalle de confiance à $95 \%: 0,34$ à 8,22), la gestion anesthésique, les délais de récupération ou la douleur postopératoire entre les patients roux et les patients témoins. Il y a eu des différences significatives dans les relations entre les concentrations d'anesthésiques volatils pharmacocinétiquement stables et les valeurs de l'indice bispectral entre les patients roux et les témoins $(P<0,001)$, mais sans implications cliniques.

D. Glick, MD

Department of Anesthesia \& Critical Care, University of Chicago, Chicago, IL, USA

G. A. Mashour, $\mathrm{MD}, \mathrm{PhD}$

Department of Anesthesiology, University of Michigan Medical School, Ann Arbor, MI, USA
Conclusion Il n'y a pas eu de différences démontrables entre les patients roux et les témoins sur la réponse aux agents anesthésiques et analgésiques, ou sur les paramètres de récupération. Ces constatations suggèrent que la gestion anesthésique et analgésique périopératoire ne doit pas être modifiée en se basant sur le seul phénotype autodécrit de cheveux roux.

Genetic factors manifesting as the red-hair phenotype are hypothesized to impact anesthetic and analgesic sensitivity. ${ }^{1-3}$ With rare exceptions, the red-hair phenotype is produced by homozygous loss-of-function mutations of the melanocortin-1 receptor (MC1R) gene. ${ }^{4-6}$ Deletion of MC1R in a murine model results in an increased requirement of volatile anesthesia to suppress movement in response to noxious mechanical stimuli. ${ }^{7,8}$ Although MC1R primarily regulates expression of pigment molecules in the skin and hair, ${ }^{9}$ it is also expressed in low levels in the central nervous system (CNS), including glial cells, pituitary cells, and periaqueductal gray matter. ${ }^{10-12}$ Periaqueductal gray matter plays a role in antinociception and is active in response to painful stimuli. ${ }^{13,14}$ Although the function of MC1R in the CNS is currently unknown, its expression in neural regions, which may modulate anesthetic and analgesic sensitivity, offers a potential mechanism for these observations.

Several animal and non-clinical human studies provide preliminary evidence suggesting that the red-hair phenotype is associated with decreased sensitivity to the effects of anesthetic agents ${ }^{1-3,7,8}$; however, one non-clinical human study failed to find a difference in anesthetic sensitivity. ${ }^{15}$ A clinical study conducted on this phenomenon found no difference in postoperative recovery time, pain, and quality of recovery for the redhair phenotype. ${ }^{16}$ There is a paucity of large clinical trials concurrently evaluating anesthetic management, anesthetic sensitivity, and postoperative outcomes. Individuals with decreased sensitivity to anesthetic agents may be at risk for receiving inadequate anesthesia and experiencing subsequent sequelae; the most concerning consequence would be intraoperative awareness with postoperative recall (AWR). Clarifying the role of hair colour in anesthetic sensitivity and the resulting clinical manifestations would better guide clinical management of patients with red hair. In the BAG-RECALL clinical trial registration (www.clinicaltrials.gov; NCT00682825), a pre-specified secondary analysis was exploring the effect of red-hair phenotypes on the relationship between end-tidal anesthetic concentrations (ETAC) and the bispectral index (BIS) as a surrogate for depth of anesthesia. In this matched historical cohort study, we pursued this pre-specified analysis in addition to other post hoc analyses. We evaluated 
whether red-haired patients 1) are at an increased risk for AWR, 2) receive more anesthetic and analgesic agents, and 3) require more volatile anesthetic agents when compared with patients without red hair. We also explored the association between the red-hair phenotype and several recovery variables.

\section{Methods}

\section{Subjects}

This matched historical cohort study is a secondary analysis of data from the BIS or Anesthetic Gas to Reduce Explicit Recall (BAG-RECALL) trial. ${ }^{17}$ The BAG-RECALL trial was a prospective international multicentre randomized controlled trial conducted in three academic centres in the United States (Washington University, St. Louis, MO; University of Chicago, IL) and in Canada (University of Manitoba, Winnipeg, MB). The trial enrolled 6,041 surgical patients at high risk for AWR from May 2008 to May 2010. Patients were excluded from the study if they had dementia, were unable to provide written informed consent, or had a history of stroke with residual neurologic deficits. Recruited patients were $18 \mathrm{yr}$ or older, scheduled for elective surgery under general anesthesia, and had at least one risk factor for AWR. Participating patients were randomly assigned to either an electroencephalogram-derived BIS-guided anesthesia protocol or an ETAC-guided anesthesia protocol. The main purpose of the parent trial was to assess whether a BIS-guided protocol was superior to an ETAC-guided protocol for the prevention of AWR. In the BIS group, an audible alarm was set to indicate when the BIS value exceeded 60 or fell below 40. No ETAC alarms were set in the BIS group, and there was no recommendation to maintain the ETAC within a specific range. In the ETAC group, an audible alarm was set to indicate when the ETAC fell below 0.7 or exceeded a 1.3 ageadjusted minimum alveolar concentration (aaMAC). In the event that alarm settings were unavailable for ETAC, alarms were set for inspired anesthetic agents. A sign was affixed to the anesthesia machines reminding practitioners to check the BIS value or the ETAC and to consider whether the patient might be aware. The BIS and ETAC protocols did not prescribe or restrict the use of anesthetic agents. ${ }^{17}$

Of the 5,713 patients assessed for AWR, 319 patients reported having natural red hair. Using a publically available propensity score-matching procedure written in $\mathrm{SAS}^{\circledR}$ code and referred to as the "greedy" matching algorithm, ${ }^{18}$ red-haired patients were matched with five controls from the remaining patients by age, body mass index (BMI), race, sex, and American Society of Anesthesiologists (ASA) physical status. The greedy matching algorithm is an iterative process based on a rank function used to find the "best" (the closest) match utilizing specified criteria. A 5:1 ratio was chosen to increase the probability that sufficient numbers of patients with AWR would be available in the matched sample to allow for meaningful analysis. Additionally, several studies $^{19-21}$ have shown that a $5: 1$ propensity score matching ratio with reasonably acceptable caliper can be beneficial in terms of reducing bias and providing stable estimates. The purpose of the greedy match was to achieve groups that were approximately similar at baseline.

Data for the BAG-RECALL trial were recorded at minimum intervals of one minute by means of MetaVision software $\left(\mathrm{iMDsoft}^{\circledR}\right.$, Needham, MA, USA), direct electronic transfer of data to Microsoft ${ }^{\circledR}$ Excel $^{\circledR}$ (Microsoft, Redmond, WA, USA), or direct electronic transfer of data with the use of TrendFace Solo software (ixellence GmBH, Wildau, Germany). Manual records of anesthesia and digital photographs of monitor trends were used as alternative sources of data in the rare instances that the computer data or the electronic anesthesia records were incomplete. Where capture of perioperative information was not possible, cases were deleted for each specific analysis separately as described below. Additional data regarding red hair and other related information were collected using a simple questionnaire interview.

\section{Outcomes measured}

\section{Awareness}

Upon enrolment, patients were assessed for risk factors for AWR. These included open heart surgery, aortic stenosis, pulmonary hypertension, ASA physical status IV, history of AWR, end-stage lung disease, history of or anticipated difficult intubation, cardiac ejection fraction $<40 \%$, marginal exercise tolerance, daily alcohol consumption, and regular use of opiates, benzodiazepines, or anticonvulsants. Patients were also asked about the presence or absence of natural red hair colour at this time. Blinded interviewers evaluated all patients for AWR using the modified Brice questionnaire ${ }^{22}$ within $72 \mathrm{hr}$ of surgery and 30 days following extubation. Reports of intraoperative memories were independently reviewed by three experts to determine whether an AWR event had occurred. When the expert opinions diverged, a fourth expert made the final determination. All definite and possible AWR events were credible intraoperative awareness experiences that did not include dreaming.

\section{Anesthetic management}

The "overall anesthetic management" was defined as the use of the following perioperative drugs: inhaled anesthetic 
agents, induction agents, sedatives, analgesics, and neuromuscular blockers. The median aaMAC was calculated for each patient from ETAC values measured during the maintenance period. ${ }^{23}$ In cases where more than one volatile anesthetic was used, concurrent aaMAC units were summed. $^{23}$ Specific details are provided in Appendix $2 .^{23}$ Total doses of intravenous induction agents, sedatives, opioid analgesics, and neuromuscular blockers given by practitioners were converted to a group representative equivalent dose for comparative analysis. Etomidate and thiopental were converted to propofol equivalents. ${ }^{24}$ Midazolam alone comprised midazolam equivalents. Fentanyl, hydromorphone, methadone, sufentanil, and meperidine were converted to morphine equivalents. ${ }^{25}$ Atracurium, mivacurium, pancuronium, and rocuronium were converted to vecuronium equivalents. ${ }^{24}$ For each anesthetic agent, doses within a clinically implausible range were treated as outliers and hence removed. Consolidation of anesthetic agents into classes has previously been described in the literature. ${ }^{26,27}$ Details on the consolidation approaches applied in this study are provided in Appendix 3.

\section{Anesthetic sensitivity}

To determine whether red-haired patients are less sensitive to the hypnotic effects of inhaled anesthetic agents, the relationship between anesthetic concentration (expressed as aaMAC) and depth of hypnosis (defined in this study as BIS values) was explored. Although the association between BIS and ETAC for individual patients varies considerably, a linear relationship for a population over a range of anesthesia relevant for clinical maintenance has been shown previously. ${ }^{27}$ Specific differences in this relationship between the red-haired cohort and the control cohort may provide evidence suggesting altered anesthetic requirements.

Intraoperative BIS ${ }^{\mathrm{TM}}$ values were obtained for all patients with a BIS Quatro forehead sensor and BIS XP software (Covidien; Boulder, CO, USA). As with ETAC, BIS values were recorded by MetaVision software or Trendface Solo software. Analysis of anesthesia records was confined to the maintenance period for each patient's case. The maintenance period was initiated five minutes after the BIS values fell below 60. If BIS was not available, the start of the volatile anesthesia record constituted the beginning of maintenance. The maintenance period ended when aaMAC fell below 0.2 and did not increase. If aaMAC did not drop below 0.2 , the end of the volatile anesthesia record constituted the end of maintenance.

Among the anesthesia records, measurement intervals of one second, one minute, and five minutes were used; records with one-minute intervals comprised the majority.
The BIS algorithm is dependent on $63 \mathrm{sec}$ of preceding electroencephalography (EEG) data. In order to reduce the covariance among consecutive BIS values derived from overlapping EEG data, records with one-second intervals were converted to records with one-minute intervals by selecting the first value of every minute in a given record. Records with five-minute intervals were not changed and comprised a minority of the sample.

A collection of custom scripts written in MATLAB (R) engineering software version 7.12.0, R2011a (The MathWorks Inc., Natick, MA, USA) were used to complete the conversion of ETAC to aaMAC, the evaluation of the maintenance period, the conversion from one-second to one-minute interval records, the identification of pharmacokinetically stable periods, and the calculations for equivalent anesthetic dose.

\section{Recovery characteristics}

An electronic medical record was implemented in the postoperative anesthesia care unit (PACU) at Washington University in September 2009. This allowed for additional outcomes to be automatically collected for a small subset of patients in the BAG-RECALL trial, including time to PACU discharge readiness, postoperative nausea and vomiting (PONV), and pain.

Postoperative anesthesia care unit discharge readiness was determined by an attending physician if the following criteria were satisfied: Aldrete score $\geq 9$, hemodynamic stability, no nausea or vomiting, well-controlled pain. Postoperative nausea and vomiting was determined by nurse documentation or antiemetic administration. Finally, the numerical rating scale assessed pain every ten minutes while the patient was in the PACU. Severe pain was defined as a score of $\geq 8$ during the initial sixty minutes. The methods and comparative analysis of postoperative outcomes are available for all subjects by treatment arm of the primary trial. ${ }^{28}$ This study includes a comparative analysis of postoperative outcomes in regard to the redhaired and matched control cohorts from this small subset.

Statistical analysis

\section{Awareness}

Baseline characteristics of red-haired patients were compared with those of patients without red hair. Categorical variables were compared using Chi square tests, and normally distributed continuous variables (by the standard univariate criteria of normality: skewness and kurtosis) were compared with the Student's $t$ test for independent samples. A greedy match algorithm with the minimum caliper range was applied to reduce the effects of 
potential confounding factors in the sample and to provide stable estimates. ${ }^{19-21}$ We used the greedy iterative matching process ${ }^{18}$ to identify five control patients for each red-haired patient based on the following variables: age, BMI, race, sex, and ASA physical status. The ASA physical status, a categorical variable, ranged from I-IV, with larger numbers indicative of more severe systemic illness. Age was incorporated as a continuous measure, and BMI was categorized according to World Health Organization (WHO) definitions. All variables were matched exactly, with the exception of ASA physical status where a caliper of 1 was allowed. To ensure that the red-haired cohort and the resulting control cohort had balanced baseline characteristics, comparisons were repeated between cohorts after matching. A two-sided Fisher's exact test was used to calculate the relative risk (RR) $95 \%$ confidence interval (CI) of AWR in the redhaired $v s$ the non-red-haired matched cohort.

\section{Anesthetic management}

Overall anesthetic management between cohorts was compared using Hotelling's $\mathrm{T}^{2}$, a multivariate $t$ test. This statistical analysis allows concurrent comparisons of related dependent variables between groups. Median aaMAC and weight-adjusted midazolam, propofol, morphine, and vecuronium equivalents were included from each case. ${ }^{2-25}$ The Hotelling's $\mathrm{T}^{2}$ requires that individual variables are normally distributed and that the condition of multivariate normality is met. In order to achieve normal distributions, Box-Cox transformations were applied to all anesthetic agents within the matched cohorts, excepting the aaMAC values. Following transformation, patients found to be multivariate outliers were excluded from the analysis.

\section{Anesthetic sensitivity}

Anesthetic sensitivity was conceptualized according to the response of the brain to increasing anesthetic concentration. As this response cannot be measured clinically after loss of responsiveness, the BIS was used as a surrogate response variable. The output of the BIS is a numerical index with values approaching 100 that reflect brain arousal and values approaching zero that signify electrical cortical brain suppression. Generally, the BIS decreases in a population with increasing volatile anesthetic concentration, ${ }^{27}$ and the BIS is used clinically as a crude metric of anesthetic depth. The relationship between anesthetic concentration as the independent variable and BIS as the dependent variable can therefore be used as a crude measure of anesthetic sensitivity. A linear mixed-effects model with an exponential correlation structure was implemented with
BIS as the primary outcome. The main predictors of BIS were ETAC (measured in aaMAC units) and red-hair phenotype (categorical variable: yes or no). Repeated measure analysis of ETAC included only steady state values where ETAC was within plus or minus 0.05 aaMAC units for the preceding ten minutes. This censoring of nonsteady-state ETAC values was performed to reduce pharmacokinetic confounding in the analysis of the BIS $v s$ ETAC relationship. Additionally, the following covariates of BIS previously found to be statistically significant were included: age $<60$, sex, ASA physical status $>$ III, nitrous oxide administration, midazolam dosing $>2 \mathrm{mg}$, and morphine equivalents $>50 \mathrm{mg} .{ }^{27}$ The BIS and ETAC protocol assignments were not included as variables in this model as red-haired and control patients were split evenly between them, and in the parent trial, anesthetic management was not found to be different between the protocols. A random effect was included for the intercept to allow for variation between patients. An interaction coefficient for aaMAC and red-hair was added to compare differences in the relationship between aaMAC and BIS between cohorts. The average regression lines in the model between the red-haired and control groups were compared with Student's $t$ test. We used the Bayesian information criterion measure of model adequacy to test the contribution of the red-hair parameter to the overall fit of the model.

\section{Recovery characteristics}

For patients who were admitted to the PACU from the operating room (OR), time to PACU discharge readiness was compared between cohorts with Kaplan-Meier analysis. Similarly, for patients who were admitted directly to the intensive care unit (ICU) from the OR, ICU length of stay was compared between cohorts with Kaplan-Meier analysis. The incidence of PONV and severe postoperative pain in the PACU were compared between the cohorts with Chi square tests.

All above statistical analyses were performed using SPSS ${ }^{\circledR}$ Statistics version 19 (IBM Corporation, Somers, NY, USA) and SAS ${ }^{\circledR} 9.2$ (SAS Institute Inc., Cary, NC, USA). Values of $P<0.05$ were considered to be statistically significant. All reported $P$ values are twosided.

\section{Results}

Patients

Of the 6,041 patients enrolled in the BAG-RECALL clinical trial, 5,731 were evaluated for AWR, and there were $319(5.57 \%)$ patients with natural red hair. These 
patients were matched 1:5 with non-red-haired controls $(n=1,595)$ for a study population of 1,914 patients. Prior to matching, the red-haired cohort included more patients who were female, white, and had a higher ASA physical status (Table 1). Sex and ASA physical status have been shown to affect the BIS-ETAC relationship and were balanced between the red-hair cohort and the matched control cohort (Table 1).

\section{Awareness}

No patients were excluded from this analysis. As determined by expert review, two patients in the redhaired cohort $(2 / 319$ or $0.63 \%)$ had definite or possible AWR, and six patients in the matched control cohort (6/ 1,595 or $0.38 \%$ ) had definite or possible AWR. After matching, there was no significant difference in the RR of AWR between red-haired patients and control patients $(\mathrm{RR}=1.67 ; 95 \%$ CI 0.34 to 8.22$)$.

\section{Anesthetic management}

The drug categories and dosing equivalents included in the anesthetic management of the red-haired cohort and matched control cohort are presented in Table 2. The median aaMAC values were calculated from repeated ETAC measurements for each individual case, and the mean aaMAC values (SD) included in Table 2 summarize the median values for each cohort. There were 34 patients missing from the red-haired cohort (10.7\%), and 146 patients missing from the matched control cohort $(9.2 \%)$. Ten patients $(0.5 \%)$ were found to be multivariate outliers and considered inconsistent with normal distribution of the sample. These patients were removed and counted among the missing patients. Collectively, there was no difference in the anesthetic management of the red-haired cohort $(n=285)$ compared with the matched control cohort $(n=1,449) \quad\left(\mathrm{F} \quad(5,1728)=0.91 ;\right.$ Hotelling's $\mathrm{T}^{2}=4.54$; $P=0.476)$.

\section{Anesthetic sensitivity}

After pharmacokinetic censoring (exclusion of non-steadystate ETAC values, i.e., where ETAC was not within \pm 0.05 aaMAC units for the preceding ten minutes), there were 80 patients missing from the red-haired cohort $(25.1 \%)$ and 332 patients missing from the matched control cohort (20.8\%). The estimates, standard errors, $95 \% \mathrm{CI}$, and $P$ values for the linear mixed-model parameters are presented in Table 3 . The Figure is a scatter plot of all intraoperative BIS and ETAC values for the study population with colour coding by cohort. Best fit lines for the red-haired cohort and the matched control cohort overlay the scatterplot. The relationship between the pharmacokinetically stable volatile anesthetic concentrations and the BIS values in the red-haired cohort was statistically significantly different from that of the matched control cohort $(P<0.001)$, but with clinically insignificant implications. The Bayesian information criteria for the model variable with and without the red-hair were $474,890.4$ and $474,875.3$, respectively. The difference between these models was very small (15.1 points or $0.0032 \%$ ), implying that the addition of the redhair variable minimally explained the variance of the model.

\section{Recovery characteristics}

Of patients who were directly admitted to the PACU, 575 (10.1\%) provided data regarding time to PACU discharge readiness. Comparison with the log-rank test showed that there was no significant difference between the red-haired cohort $(n=90)$ and the matched control cohort $(n=485)$ $\left(\chi^{2}=1.98 ; P=0.16\right)$. Patients admitted to the PACU after implementation of an electronic medical record in September 2009 were assessed for PONV and postoperative pain. In the 243 patients assessed for PONV, nine patients in the red-haired cohort $(22.0 \%)$ and 38 patients in the control cohort (18.8\%) suffered from PONV. There was no significant association between the red-hair phenotype and the occurrence of PONV $\left(\chi^{2}=0.22 ; P=0.64\right)$. In the 235 patients with pain measurements, 11 patients in the red-haired cohort $(28.9 \%)$ and 71 patients in the control cohort $(36.0 \%)$ experienced severe pain in the PACU. The difference in the incidence of severe pain was not significant $\left(\chi^{2}=0.71 ; P=0.40\right)$. Of the patients admitted to the ICU, 831 (14.5\%) provided data regarding length of stay. Comparison with log-rank test showed that there were no significant differences between the red-haired cohort $(n=153)$ and the matched control cohort $(n=678)\left(\chi^{2}=0.40 ; P=0.526\right)$.

\section{Discussion}

In this large matched historical cohort analysis, the red-hair phenotype was not associated with alterations in AWR, anesthetic management, anesthetic sensitivity, or recovery from general anesthesia. These findings are clinically important and contrast with published hypothesisgenerating animal research and small proof-of-principle human studies.

\section{Awareness}

Conceptually, decreased anesthetic sensitivity in this population could result in insufficient anesthesia and potentially even AWR. Nevertheless, there is a lack of 
Table 1 Characteristics of the red-haired cohort and the unmatched and matched non-red-haired control cohorts

\begin{tabular}{|c|c|c|c|c|c|}
\hline & $\begin{array}{l}\text { Red Hair } \\
(n=319)\end{array}$ & $\begin{array}{l}\text { Controls } \\
(n=5,394)\end{array}$ & $P$ Value* & $\begin{array}{l}\text { Matched Controls } \\
(n=1,595)\end{array}$ & $P$ Value $^{\dagger}$ \\
\hline Age (yr) - mean (SD) & $59.7(13.5)$ & $59.7(14.3)$ & 0.931 & $59.2(14.8)$ & 0.613 \\
\hline Male $-n(\%)$ & $157(49.2)$ & $3,145(58.3)$ & 0.001 & $785(49.2)$ & 1.000 \\
\hline BMI $\left(\mathrm{kg} \cdot \mathrm{m}^{-2}\right)-$ mean $(\mathrm{SD})$ & $30.0(8.0)$ & $29.8(8.1)$ & 0.697 & $29.7(8.4)$ & 0.565 \\
\hline Race $-n(\%)$ & & & $<0.001$ & & 1.000 \\
\hline White & $308(96.6)$ & $4,486(83.2)$ & & $1,540(96.6)$ & \\
\hline Black & $7(2.2)$ & 719 (13.3) & & $35(2.2)$ & \\
\hline Other & $4(1.3)$ & $189(3.5)$ & & $20(1.3)$ & \\
\hline ASA physical status $-n(\%)$ & & & 0.041 & & 0.968 \\
\hline I & $3(0.9)$ & $39(0.7)$ & & $11(0.7)$ & \\
\hline II & $54(16.9)$ & $821(15.2)$ & & $271(17.0)$ & \\
\hline III & $133(41.7)$ & $2,690(49.9)$ & & $674(42.3)$ & \\
\hline IV & $129(40.4)$ & $1,844(34.2)$ & & $639(40.1)$ & \\
\hline \multicolumn{6}{|l|}{ Comorbid conditions $-n(\%)$} \\
\hline Coronary disease & $114(35.7)$ & $1,851(34.3)$ & 0.604 & $527(33.0)$ & 0.352 \\
\hline CVA/stroke & $11(3.4)$ & $154(2.9)$ & 0.539 & $40(2.5)$ & 0.341 \\
\hline Congestive heart failure & $37(11.6)$ & $667(12.4)$ & 0.686 & $186(11.7)$ & 0.975 \\
\hline COPD & $34(10.7)$ & $650(12.1)$ & 0.457 & $212(13.3)$ & 0.200 \\
\hline Dysrhythmias & $31(9.7)$ & $649(12.0)$ & 0.215 & $211(13.2)$ & 0.085 \\
\hline \multicolumn{6}{|l|}{ Diabetes mellitus } \\
\hline Oral hypoglycemic / no insulin & $45(14.1)$ & $958(17.8)$ & 0.096 & $243(15.2)$ & 0.607 \\
\hline \pm Oral hypoglycemic / insulin & $22(6.9)$ & $287(5.3)$ & 0.227 & $88(5.5)$ & 0.334 \\
\hline Hypertension & $190(59.6)$ & $3,273(60.7)$ & 0.691 & $903(56.6)$ & 0.332 \\
\hline Previous coronary vascularization & $16(5.0)$ & $381(7.1)$ & 0.162 & $95(6.0)$ & 0.512 \\
\hline Peripheral vascular disease & 37 (11.6) & $506(9.4)$ & 0.189 & $147(9.2)$ & 0.188 \\
\hline Sleep apnea & $35(11.0)$ & $699(13.0)$ & 0.303 & $189(11.8)$ & 0.656 \\
\hline Valvular heart disease & $65(20.4)$ & $1,052(19.5)$ & 0.702 & $330(20.7)$ & 0.900 \\
\hline History of vascular surgery & $15(4.7)$ & $281(5.2)$ & 0.691 & $83(5.2)$ & 0.711 \\
\hline Liver disease & $12(3.8)$ & $208(3.9)$ & 0.932 & $58(3.6)$ & 0.913 \\
\hline \multicolumn{6}{|l|}{ Composite number of preexisting conditions } \\
\hline Median (IQR) & $2(2)$ & $2(2)$ & 0.297 & $2(2)$ & 0.985 \\
\hline \multicolumn{6}{|l|}{ Trial Protocol $-n(\%)$} \\
\hline BIS Guided & $156(48.9)$ & $2,705(50.1)$ & 0.666 & $811(50.8)$ & 0.526 \\
\hline ETAC Guided & $163(51.1)$ & $2,689(49.9)$ & & $784(49.2)$ & \\
\hline \multicolumn{6}{|l|}{ Inclusion criteria met for primary study $-n(\%)$} \\
\hline Planned heart surgery & $122(38.2)$ & $1,919(35.6)$ & 0.334 & $596(37.4)$ & 0.768 \\
\hline Aortic stenosis & $34(10.7)$ & $488(9.0)$ & 0.332 & $163(10.2)$ & 0.814 \\
\hline Pulmonary hypertension & $12(3.8)$ & $259(4.8)$ & 0.396 & $94(5.9)$ & 0.129 \\
\hline Regular opiate use & $89(27.9)$ & $1,377(25.5)$ & 0.346 & $454(28.5)$ & 0.838 \\
\hline Regular benzodiazepine use & $59(18.5)$ & $840(15.6)$ & 0.164 & $320(20.1)$ & 0.521 \\
\hline Regular Cocaine use & $1(0.3)$ & $32(0.6)$ & 0.522 & $10(0.6)$ & 0.499 \\
\hline Regular Anticonvulsants & $28(8.8)$ & $395(7.3)$ & 0.335 & $118(7.4)$ & 0.397 \\
\hline Daily alcohol use & $46(14.4)$ & $841(15.6)$ & 0.575 & $227(14.2)$ & 0.930 \\
\hline ASA physical status IV & $129(40.4)$ & $1,844(34.2)$ & 0.022 & $639(40.1)$ & 0.900 \\
\hline End-stage lung disease & $10(3.1)$ & $106(2.0)$ & 0.15 & $45(2.8)$ & 0.760 \\
\hline History of intraoperative awareness & $9(2.8)$ & $82(1.5)$ & 0.071 & $29(1.8)$ & 0.241 \\
\hline History of or anticipated difficult intubation & $28(8.8)$ & $440(8.2)$ & 0.695 & $116(7.3)$ & 0.352 \\
\hline Cardiac ejection fraction $<40 \%$ & $26(8.2)$ & $485(9.0)$ & 0.609 & $135(8.5)$ & 0.854 \\
\hline
\end{tabular}


Table 1 continued

\begin{tabular}{lcccc}
\hline & $\begin{array}{l}\text { Red Hair } \\
(n=319)\end{array}$ & $\begin{array}{l}\text { Controls } \\
(n=5,394)\end{array}$ & $P$ Value* & $\begin{array}{l}\text { Matched Controls } \\
(n=1,595)\end{array}$ \\
\hline $\begin{array}{l}\text { Marginal exercise tolerance } \\
\text { Composite number of inclusion criteria met } \\
\text { Median (IQR) }\end{array}$ & $144(45.1)$ & $2,506(46.5)$ & 0.646 & $727(45.6)$ \\
\hline
\end{tabular}

*Comparative analysis between the red-haired cohort and non-red-haired cohort

$\dagger$ Comparative analysis between the red-haired cohort and matched non-red-haired cohort

$P$ values were calculated using Chi square tests for categorical variables, independent samples $t$ test for continuous variables, and Wilcoxon ranksum test for composite values

$\mathrm{BMI}=$ body mass index; $\mathrm{ASA}=$ American Society of Anesthesiologists; $\mathrm{CVA}=$ cerebrovascular accident; COPD $=$ chronic obstructive pulmonary disease; IQR = interquartile range; $\mathrm{BIS}=$ bispectral index; ETAC $=$ end-tidal anesthetic concentrations

Table 2 Drug categories and dosing equivalents included in the anesthetic management of the red-hair cohort and matched control cohort

\begin{tabular}{lcc}
\hline & Red Hair $(n=285)$ & Non Red Hair $(n=1,449)$ \\
\hline aaMAC $^{*}$ & $0.92(0.18)$ & $0.91(0.18)$ \\
Midazolam equivalents $^{\dagger}$ & $0.028(0.026)$ & $0.028(0.026)$ \\
Morphine equivalents $^{\dagger}$ & $0.80(1.15)$ & $0.75(0.85)$ \\
Propofol equivalents $^{\dagger}$ & $2.11(2.00)$ & $2.22(1.75)$ \\
Vecuronium equivalents $^{\dagger}$ & $0.12(0.11)$ & $0.12(0.09)$ \\
\hline
\end{tabular}

Values are expressed as mean (SD)

*Mean (SD) of the median age-adjusted minimum alveolar concentration (aaMAC) during anesthetic maintenance. ${ }^{\dagger}$ Doses are reported in $\mathrm{mg} \cdot \mathrm{kg}^{-1}$

Table 3 Parameter estimates for the linear mixed effects model evaluating the relationship between age-adjusted minimum alveolar anesthetic concentration (aaMAC) and bispectral index (BIS)

\begin{tabular}{|c|c|c|c|c|}
\hline Parameter & Estimate & Standard Error & $95 \% \mathrm{CI}$ & $P$ Value \\
\hline Extrapolated intercept & 61.32 & 1.05 & 61.25 to 61.38 & $<0.0001$ \\
\hline aaMAC & -15.55 & 0.42 & -15.58 to -15.53 & $<0.0001$ \\
\hline Red-hair & -3.90 & 1.10 & -3.97 to -3.83 & 0.0004 \\
\hline aaMac * Red-hair interaction & 3.47 & 1.06 & 3.40 to 3.53 & 0.0011 \\
\hline Age $<60$ yr & -3.63 & 0.42 & -3.66 to -3.61 & $<0.0001$ \\
\hline Female & -1.46 & 0.41 & -1.49 to -1.44 & 0.0003 \\
\hline ASA physical status $>$ III & 1.29 & 0.45 & 1.26 to 1.32 & 0.004 \\
\hline $\mathrm{N}_{2} \mathrm{O}$ not used & -2.52 & 0.93 & -2.58 to -2.46 & 0.0066 \\
\hline Midazolam $>2 \mathrm{mg}$ & -0.92 & 0.45 & -0.95 to -0.90 & 0.0413 \\
\hline Morphine equivalents $>50 \mathrm{mg}$ & -1.82 & 0.44 & -1.85 to -1.80 & $<0.0001$ \\
\hline
\end{tabular}

Overall model fit statistics: -2 Res Log Likelihood $=1261535$

Likelihood Ratio Test Chi square $=293983.83$ with $P<0.0001$ (1 DF). CI $=$ confidence interval; ASA $=$ American Society of Anesthesiologists

prior studies evaluating this important functional outcome. The current study shows no difference in the incidence of AWR between patients with red hair and those without red hair. The limitation of this finding is that with low numbers of patients with AWR in each cohort, the confidence interval is wide and does not exclude up to an eightfold increased RR of AWR attributable to red hair.
Although this secondary analysis was underpowered to detect a small difference in the incidence of AWR attributable to red hair, based on a sensitivity analysis, it was reasonably powered to detect a clinically important tenfold effect size. For example, assuming a two-tailed test with 0.002 incidence of AWR in the non-red-haired (control) group, a significance level $\alpha=0.05$, a power of 
$80 \%$, and using the actual sample sizes of 319 red-haired patients and 1,595 non-red-haired patients, the smallest difference in incidence of AWR that can be detected is 0.016, which corresponds with a 0.018 incidence of AWR among red-haired patients.

\section{Anesthetic management}

Despite evidence supporting an association between the red-hair phenotype and decreased anesthetic sensitivity, the current study found no difference in the median volatile anesthetic and total doses of analgesics, sedatives, induction agents, and neuromuscular blocking agents administered between cohorts. This suggests that practitioners have not interpreted the findings from animal and human studies to suggest that a change in anesthetic management is required. These previous studies have shown decreased sensitivity to analgesic and anesthetic agents with MC1R genotype or with red-hair phenotype. ${ }^{1-3,7,8}$ Nevertheless, this finding based on practice at three academic centres in North America cannot exclude that there are practitioners elsewhere who do systematically alter their anesthetic management for red-haired patients.

\section{Anesthetic sensitivity}

In a study of healthy female volunteers, subjects with red hair required 19\% more desflurane than those with dark hair to suppress movement in response to noxious electrical stimuli. ${ }^{1}$ This increased anesthetic requirement associated with the red-hair phenotype has not been replicated in a surgical population. A prospective matched cohort study of surgical patients by Myles et al. found no difference in the anesthetic requirements between red-haired patients and control patients; the red-haired and control patients received similar amounts of volatile anesthetic and had comparable average BIS values. ${ }^{16}$ As aaMAC and BIS values fluctuate intraoperatively, important information is lost by simply reporting average values. To evaluate sensitivity to volatile anesthetics with additional granularity, the current study utilized a mixed-effects model. This statistical technique models hypnotic depth (BIS) as a function of volatile anesthetic concentration (ETAC) over pharmacokinetically stable epochs during anesthetic maintenance. The model showed a statistically significant difference between the red-haired cohort and control cohort, which is not likely to be clinically significant. The lack of a clinically important difference can be visually appreciated from the Figure where the regression lines actually intersect within the therapeutic range of ETAC. One of the factors contributing to the statistically significant finding was simply the very large

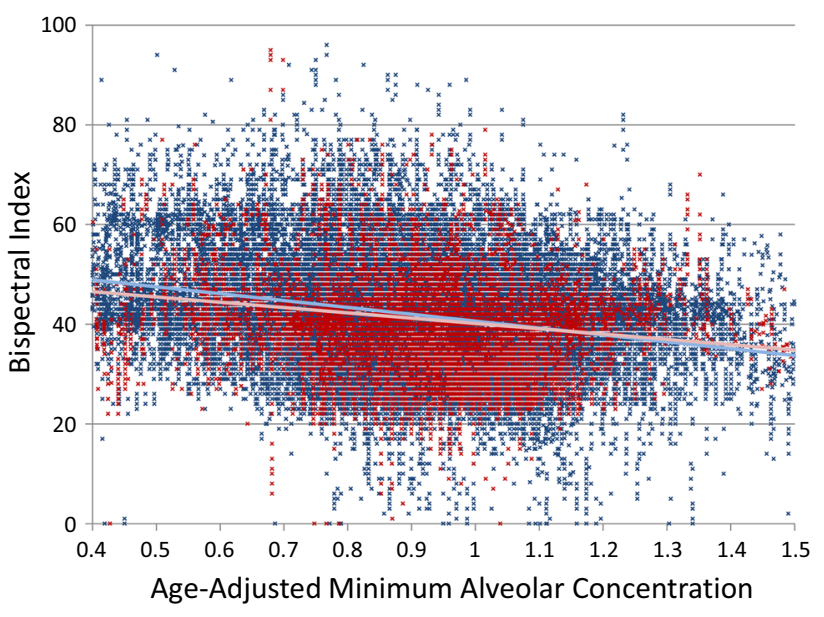

Figure Scatterplot of age-adjusted minimum aveolar anesthetic concentrations during the anesthetic maintenance phase plotted against corresponding bispectral index values for patients with natural red hair (red crosses) and for matched controls (blue crosses). Linear mixed-effects model for patients with natural red hair (red line) and for matched controls (blue line) are superimposed

sample size. The major limitation of this finding is that the BIS is a surrogate measure of anesthetic depth without a firm neurobiological foundation. Thus, based exclusively on this metric, we cannot truly rule out that red-haired patients require increased anesthetic concentrations to achieve comparable anesthetic endpoints with their nonred-haired counterparts. Nonetheless, even if there is a difference in anesthetic requirement, it is probably not substantial based on the fact that there was no significantly increased incidence of AWR in the red-haired population.

\section{Recovery characteristics}

The results of several other studies, including studies in healthy volunteers, suggest that the red-hair phenotype is associated with decreased sensitivity to sedative and analgesic medications. ${ }^{2,3}$ In a randomized placebocontrolled crossover trial, midazolam administration produced less sedation and cognitive impairment in redhaired subjects than in control subjects. ${ }^{2}$ Red-haired female volunteers showed increased sensitivity to thermal pain and decreased efficacy of the local anesthetic agent, lidocaine. ${ }^{3}$ While these results suggest patients with red hair may recover from anesthesia faster and suffer more severe postoperative pain than those without red hair, a study in surgical patients did not find any difference in the time to recovery after surgery or the severity of postoperative pain. ${ }^{16}$ The results of the present study show no difference between cohorts for PONV as well as reinforce the previous findings of no difference between cohorts in time to recovery, ICU length of stay, and postoperative pain. 
Further limitations

Patients reported to researchers whether or not they had natural red hair. There could have been mistakes in these reports, and the phenotype does not necessarily confirm the genotype. It therefore remains possible that this study missed clinically important effects of the MC1R genotype on anesthesia requirements and recovery characteristics. Patients in this study were originally recruited for being at high risk for experiencing AWR. Patients at high risk for AWR may have differences in their anesthetic management, anesthetic requirement, and incidence of AWR. The findings in this study may therefore not be generalizable to unselected surgical patients. With respect to anesthetic requirement, multiple different inhalational agents, intravenous agents, analgesics, and muscle relaxants were used in the current study. The methodological approach relied on conversion to aaMAC, midazolam, propofol, morphine, and vecuronium equivalents to compare drug administration between the two groups. This approach is inexact, and conversion to equivalents across multiple patient populations and drug combinations is susceptible to potential error.

\section{Conclusion}

In conclusion, the red-hair phenotype does not appear to alter risk of AWR, anesthetic requirements, or recovery characteristics in surgical patients. These results do not support past animal and human volunteer studies, but they are consistent with a recent study of surgical patients and add significantly to the literature by showing no significant increase in AWR, the functional outcome of decreased anesthetic sensitivity. Based on these findings, red-haired patients can be reassured that there do not appear to be important differences in their anesthetic and analgesic requirements for surgery, and anesthesiologists should not alter their management based on the self-reported redhaired phenotype alone.

Acknowledgements This publication was made possible by Grants UL1 TR000448 and RR024992, and TL1 TR000449 and RR024995 from the National Center for Advancing Translational Sciences (NCATS) and the National Center for Research Resources (NCRR), National Institutes of Health, Bethesda, Maryland. The content is solely the responsibility of the authors and does not necessarily represent the official views of the NIH. The BAG-RECALL trial was supported by a grant (CFM-08/15/2007-Avidan) awarded by the Foundation for Anesthesia Education and Research, Rochester, Minnesota; and the American Society of Anesthesiologists, Park Ridge, Illinois; as well as institutional and departmental sources. Analytical and informatics resources as well as research mentorship for this study were provided by the Institute of Quality Improvement, Research and Informatics (INQUIRI) at Washington University in St. Louis, Missouri.
Conflicts of interest None declared.

\section{Appendix 1}

Modified Brice Questionnaire ${ }^{19}$

1 What was the last thing your remember before going to sleep?

2 What is the first thing you remember after waking up?

3 Do you remember anything between going to sleep and waking up?

4 Did you dream during your procedure?

5 What was the worst thing about your operation?

\section{Appendix 2}

$\mathrm{MAC}_{\text {Age }}=\mathrm{MAC}_{40} * 10^{(\mathrm{Age}-40) *(-0.00269)}$

Age-adjusted minimum aveolar concentration (aaMAC or $\left.\mathrm{MAC}_{\mathrm{Age}}\right)$ is calculated from the minimum aveolar concentration (MAC) at age 40 for a given volatile anesthetic at sea level (sevoflurane $=1.8 \%$, isoflurane $=$ $1.17 \%$, desflurane $=6.6 \%$, and nitrous oxide $=104 \%$ ). When more than one agent was administered concurrently, the net aaMAC value was calculated as the sum of the aaMAC values for the individual agents. ${ }^{23}$

\section{Appendix 3}

Anesthetic drugs, equivalence drugs, equivalence factors, and dose limits

\begin{tabular}{lllr}
\hline Drug & $\begin{array}{l}\text { Equivalence } \\
\text { Drug }\end{array}$ & $\begin{array}{l}\text { Equivalence } \\
\text { Factor* }\end{array}$ & $\begin{array}{r}\text { Upper Dose } \\
\text { Limit }^{\dagger}(\mathrm{mg})\end{array}$ \\
\hline Midazolam & Midazolam & 1 & 100 \\
Morphine & Morphine & 1 & 200 \\
Fentanyl & Morphine & 100 & 20 \\
Hydromorphone & Morphine & 5 & 20 \\
Methadone & Morphine & 0.125 & 100 \\
Sufentanil & Morphine & 1000 & 10 \\
Meperidine & Morphine & 0.1 & 300 \\
Propofol & Propofol & 1 & 6,200 \\
Etomidate & Propofol & 5.6 & 50 \\
Thiopental & Propofol & 0.5 & 2,000 \\
Vecuronium & Vecuronium & 1 & 50 \\
Atracurium & Vecuronium & 0.248 & 500 \\
Pancuronium & Vecuronium & 0.814 & 50 \\
Rocuronium & Vecuronium & 0.190 & 250 \\
\hline
\end{tabular}

*Equivalence factor is the factor by which the Drug (column 1) is multiplied to convert it to a comparable dose of the Equivalence Drug (column 2). ${ }^{\dagger}$ Limit of the Drug dose (mg) (values beyond were excluded) 


\section{References}

1. Liem EB, Lin CM, Suleman MI, et al. Anesthetic requirement is increased in redheads. Anesthesiology 2004; 101: 279-83.

2. Chua MV, Tsueda K, Doufas AG. Midazolam causes less sedation in volunteers with red hair. Can J Anesth 2004; 51: 25-30.

3. Liem EB, Joiner TV, Tsueda K, Sessler DI. Increased sensitivity to thermal pain and reduced subcutaneous lidocaine efficacy in redheads. Anesthesiology 2005; 102: 509-14.

4. Rees JL, Flanagan N. Pigmentation, melanocortins and red hair. QJM 1999; 92: 125-31.

5. Schioth HB, Phillips SR, Rudzish R, Birch-Machin MA, Wikberg $J E$, Rees $J L$. Loss of function mutations of the human melanocortin 1 receptor are common and are associated with red hair. Biochem Biophys Res Commun 1999; 260: 488-91.

6. Healy E, Jordan SA, Budd PS, Suffolk R, Rees JL, Jackson IJ. Functional variation of MC1R alleles from red-haired individuals. Hum Mol Genet 2001; 10: 2397-402.

7. Mogil JS, Wilson SG, Chesler EJ, et al. The melanocortin-1 receptor gene mediates female-specific mechanisms of analgesia in mice and humans. Proc Natl Acad Sci U S A 2003; 100: 4867-72.

8. Xing Y, Sonner JM, Eger EI 2nd, Cascio M, Sessler DI. Mice with a melanocortin 1 receptor mutation have a slightly greater minimum alveolar concentration than control mice. Anesthesiology 2004; 101: 544-6.

9. Rees JL. Genetics of hair and skin color. Annu Rev Genet 2003; 37: 67-90.

10. Abdel-Malek ZA. Melanocortin receptors: their functions and regulation by physiological agonists and antagonists. Cell Mol Life Sci 2001; 58: 434-41.

11. Chhajlani $V$. Distribution of cDNA for melanocortin receptor subtypes in human tissues. Biochem Mol Biol Int 1996; 38 : 73-80.

12. Wikberg JE. Melanocortin receptors: perspectives for novel drugs. Eur J Pharmacol 1999; 375: 295-310.

13. Basbaum AI, Fields HL. Endogenous pain control systems: brainstem spinal pathways and endorphin circuitry. Annu Rev Neurosci 1984; 7: 309-38.

14. Hsieh JC, Stahle-Backdahl M, Hagermark O, Stone-Elander S, Rosenquist $G$, Ingvar $M$. Traumatic nociceptive pain activates the hypothalamus and the periaqueductal gray: a positron emission tomography study. Pain 1996; 64: 303-14.

15. Doufas AG, Orhan-Sungur $M$, Komatsu R, et al. Bispectral index dynamics during propofol hypnosis is similar in red-haired and dark-haired subjects. Anesth Analg 2013; 116: 319-26.
16. Myles PS, Buchanan FF, Bain CR. The effect of hair colour on anaesthetic requirements and recovery time after surgery. Anaesth Intensive Care 2012; 40: 683-9.

17. Avidan MS, Jacobsohn E, Glick D, et al. Prevention of intraoperative awareness in a high-risk surgical population. N Engl J Med 2011; 365: 591-600.

18. Parsons LS; Ovation Research Group. Reducing bias in a propensity score matched-pair sample using greedy matching techniques. Proceedings of the Twenty-Sixth Annual SAS ${ }^{\circledR}$ Users Group International Conference. Long Beach, California: SAS Institute Inc.; 2001: 214-26.

19. Austin PC. The performance of different propensity-score methods for estimating differences in proportions (risk differences or absolute risk reductions) in observational studies. Stat Med 2010; 29: 2137-48.

20. Austin PC. Statistical criteria for selecting the optimal number of untreated subjects matched to each treated subject when using many-to-one matching on the propensity score. Am J Epidemiol 2010; 172: 1092-7.

21. Rosenbaum $P R$. Design sensitivity and efficiency in observational studies. J Am Stat Assoc 2010; 105: 692-702.

22. Mashour GA, Kent C, Picton P, et al. Assessment of intraoperative awareness with explicit recall: a comparison of 2 methods. Anesth Analg 2013; 116: 889-91.

23. Nickalls $R W$, Mapleson $W W$. Age-related iso-MAC charts for isoflurane, sevoflurane and desflurane in man. Br J Anaesth 2003; 91: 170-4.

24. Cusick JM. Anesthesia \& Critical Care Reference Sheet. Goodyear, AZ, Jeffery Cusick, 2009. Available from URL: http:// anesthesiareferencesheets.com/critical-care-author.html (accessed November 2014).

25. Pereira J, Berry Otfinowski P, Hagen N, Bruera E, Fainsinger R, Summers N. Alberta Hospice Palliative Care Resource Manual, 2nd edition. Calgary, AB, Canada, Alberta Cancer Board; 2001.

26. Aranake A, Gradwohl S, Ben-Abdallah A, et al. Increased risk of intraoperative awareness in patients with a history of awareness. Anesthesiology 2013; 119: 1275-83.

27. Whitlock EL, Villafranca AJ, Lin N, et al. Relationship between bispectral index values and volatile anesthetic concentrations during the maintenance phase of anesthesia in the B-Unaware trial. Anesthesiology 2011; 115: 1209-18.

28. Fritz BA, Rao P, Mashour GA, et al. Postoperative recovery with bispectral index versus anesthetic concentration-guided protocols. Anesthesiology 2013; 118: 1113-22. 